\title{
Thyroid hormone (T3) plus dual-specificity phosphatase-5 siRNA therapy regenerates the myocardium lost as a result of doxorubicin cardiotoxicity
}

\section{Lin Tan}

Emory University School of Medicine https://orcid.org/0000-0003-0566-9064

Nikolay Bogush

Department of Medicine (Cardiology), Emory University School of Medicine

\section{Emmen Naqvi}

Department of Medicine (Cardiology), Emory University School of Medicine

\section{J Calvert}

https://orcid.org/0000-0001-6858-6042

\section{Robert Graham}

Victor Chang Cardiac Research Institute https://orcid.org/0000-0002-1042-2587

\section{W. Robert Taylor}

Emory University

\section{Nawazish Naqvi}

Emory University

\section{Ahsan Husain ( $\square$ ahusai2@emory.edu )}

Department of Medicine (Cardiology), Emory University School of Medicine https://orcid.org/00000003-3426-3469

\section{Article}

Keywords: Doxorubicin, cardiotoxicity, transient therapy, thyroid hormone, dual-specificity phosphatase-5 (DUSP5) siRNA

Posted Date: September 3rd, 2020

DOl: https://doi.org/10.21203/rs.3.rs-59095/v1

License: (c) (i) This work is licensed under a Creative Commons Attribution 4.0 International License. Read Full License 


\section{Thyroid hormone (T3) plus dual-specificity phosphatase-5 siRNA therapy regenerates the myocardium lost as a result of doxorubicin cardiotoxicity}

Lin Tan1,6, Nikolay Bogush1,6, Emmen Naqvi1, John W. Calvert2, Robert M. Graham3, Robert W. Taylor1, Nawazish Naqvi1,7 and Ahsan Husain1,7

1Department of Medicine (Cardiology), Emory University School of Medicine, Atlanta, GA, USA. 2Department of Surgery, Carlyle Fraser Heart Center, Emory University School of Medicine, Atlanta, GA, USA. 3Victor Chang Cardiac Research Institute, Darlinghurst, New South Wales, Australia.

6These authors contributed equally: Lin Tan, Nikolay Bogush.

7Address correspondence to: ahusai2@emory.edu or nnaqvi@emory.edu 


\begin{abstract}
Doxorubicin is a widely used antineoplastic drug. However, its major side effect, cardiotoxicity, results from cardiomyocyte loss that causes left ventricle (LV) wall thinning, chronic LV dysfunction and heart failure. Here, we show that transient therapy with thyroid hormone (triiodothyronine, T3) and dual-specificity phosphatase-5 (DUSP5) siRNA results in cardiomyocyte proliferation. siRNA-directed depletion of DUSP5, a nuclear localized pERK1/2-specific phosphatase, sensitizes cardiomyocytes to the proliferative effects of T3 by potentiating $\mathrm{p}$-ERK1/2 accumulation resulting from the activation of T3-mediated insulin-like growth factor-1 (IGF-1) signaling. In mice with chronic doxorubicin cardiotoxicity, this therapy regenerates the LV myocardium by cardiomyocyte proliferation, reverses LV dysfunction and prevents progressive chamber dilatation, providing a strategy for addressing a currently untreatable condition.
\end{abstract}


Anthracyclines, including doxorubicin (Adriamycin), are part of many anticancer regimens because of their potent antitumor effect1. They are used to treat a variety of malignancies, including more than 1 in 2 pediatric cancers1. However, in young cancer survivors, the relative risk of congestive heart failure is 15 times higher than in their siblings who did not have cancer2. The cumulative anthracycline dose is a major determinant of its cardiotoxicity, which results in the development of left ventricle (LV) dysfunction. Importantly, in pediatric cancer survivors, cardiovascular mortality surpasses cancer mortality3. Extant clinical trials have mainly focused on its prevention3, since once established, doxorubicin-induced cardiomyopathy is presently untreatable.

The mechanism of doxorubicin-induced cardiotoxicity involves topoisomerase-2 $\beta$ inhibition, which in cardiomyocytes causes double-stranded DNA breaks and, ultimately, many of these cells undergo p53-mediated apoptotic cell death4. In patients exposed repeatedly to doxorubicin, elevated levels indicate continuing cardiomyocyte death, whereas low serum troponin levels are associated with better outcomes5. After repeated anthracycline exposure, gradual sub-clinical LV disease is observed in many pediatric cancer survivors, which manifests as dilated cardiomyopathy, LV posterior wall (PW) thinning and reduced fractional shortening3. Therapy for this cardiomyopathy is directed at managing heart failure (e.g., diuretics and angiotensin converting enzyme inhibitors or angiotensin receptor blockers), since currently no treatments are available to directly enhance LV contractile function.

Current experimental approaches at cardiac regeneration, mostly genetic, are directed at increasing cardiomyocyte proliferation and improving LV ejection fraction when implemented at the time of, or immediately after, an acute myocardial infarct6-9. However, the vast majority of patients who are diagnosed with heart failure are those who most likely had heart failure for some time. To our knowledge, efforts to improve cardiac contractile function, through controlled 
cardiomyocyte proliferation, have not been attempted in the setting of established long-term heart failure.

A key impediment to achieving successful mouse-to-human translation is the discovery of small molecules that, when given acutely, can produce a sustained increase in LV muscle mass through controlled cardiomyocyte proliferation and, as a result, permanently reverse LV dysfunction. If possible, such an achievement would come close to a "cure" for some lethal forms of heart failure, including those arising from chronic doxorubicin-induced cardiotoxicity.

Here we describe a pharmacological strategy for stably building heart muscle by re-induction of proliferation in cardiomyocytes of uninjured adult mouse hearts, and then test its efficacy in treating experimental chronic doxorubicin-induced LV dysfunction. In neonatal cardiomyocytes, exogenous T3 stimulates insulin-like growth factor 1 (IGF-1) and IGF-1 receptor (IGF-1R) expression thereby activating extracellular signal-regulated kinases 1 and 2 (ERK1/2), which mediate cell proliferation10. In preadolescent cardiomyocytes, T3-stimulated ERK1/2 signaling is inhibited by DUSP5, a nuclear-localized p-ERK1/2-specific phosphatase11. We show that combining DUSP5 suppression with T3 therapy, for a brief period of time, produces a robust increase in p-ERK1/2 levels that induces cardiomyocyte proliferation, which we then use as a therapeutic strategy to repair hearts of young adult mice with doxorubicin-induced LV dysfunction.

\section{Results}

DUSP5 siRNA+T3 stimulates cardiomyocyte proliferation. We tested the hypothesis that combining DUSP5 suppression with T3 therapy will stimulate ERK1/2 phosphorylation and cell proliferation in mature LV cardiomyocytes. We used in vivo-jetPEI® to deliver two daily doses of DUSP5 or scrambled siRNA to $\sim 5$-week-old male mice. DUSP5 siRNA, but not scrambled 
siRNA (control), reduced DUSP5 levels in cardiomyocytes by $\sim 98 \%(P<0.001)$ (Fig. 1a). By itself, DUSP5 siRNA therapy did not increase cardiomyocyte p-ERK1/2 levels but, when supplemented with a single dose of T3 (4 ng/g, i.p.), p-ERK1/2 levels increased 2-3-fold, relative to those observed in control animals treated with scrambled siRNA+T3 $(P<0.001)$ (Fig. 1a). We examined cyclin D1 accumulation in cardiomyocytes as a readout of the ability of DUSP5 siRNA+T3 therapy to activate proliferative ERK1/2 signaling12. DUSP5 siRNA+T3 therapy increased cyclin D1 expression in LV cardiomyocytes. Scrambled siRNA, scrambled siRNA+T3 or DUSP5 siRNA monotherapy, did not (Fig. 1a).

To determine if DUSP5 siRNA+T3 therapy can stimulate cardiomyocyte proliferation, in vivo, we gave 2 daily doses of DUSP5 siRNA followed by 5 daily doses of T3 ( $2 \mathrm{ng} / \mathrm{g}$, i.p.). This T3 dosing protocol increased circulating T3 levels twofold (serum T3 levels were $3.5 \pm 0.19$ $\mathrm{ng} / \mathrm{ml}$ and $1.67 \pm 0.02 \mathrm{ng} / \mathrm{ml}$ after T3 and vehicle dosing, respectively; $P<0.001)$. Four weeks after therapy, there were $25 \%$ more ventricular cardiomyocytes in DUSP5 siRNA+T3-treated mice than in mice treated with DUSP5 siRNA alone $(P<0.001)$, or after scrambled siRNA, with or without T3 therapy ( $P<0.001$, in each case) (Fig. 1b). DUSP5 siRNA+T3-induced cardiomyocyte proliferation was accompanied by a reduction in the average cardiomyocyte cell size (by $12 \%$ for mononuclear cells, $P<0.05$, and by $15 \%$ for binuclear cells, $P<0.001$ ) (Extended Data Fig. 1).

We then used multicolor clonal analysis in double-transgenic Myh6-MerCreMer::Rosa26fsConfetti mice13 to record the proliferative strategies of individual LV cardiomyocytes after DUSP5 siRNA+T3 combination therapy. A schematic of the Confetti construct is shown in Fig. 1c. Limited Cre activation (by administration of $1.5 \mu \mathrm{g} / \mathrm{g}$ 4-hydroxytamoxifen to 3-week-old mice) resulted in cardiomyocytes being labeled with either RFP, YFP, CFP or GFP. The frequency of RFP and YFP labeling events at 8-weeks-of-age was $0.3 \%-0.8 \%$, while 
a

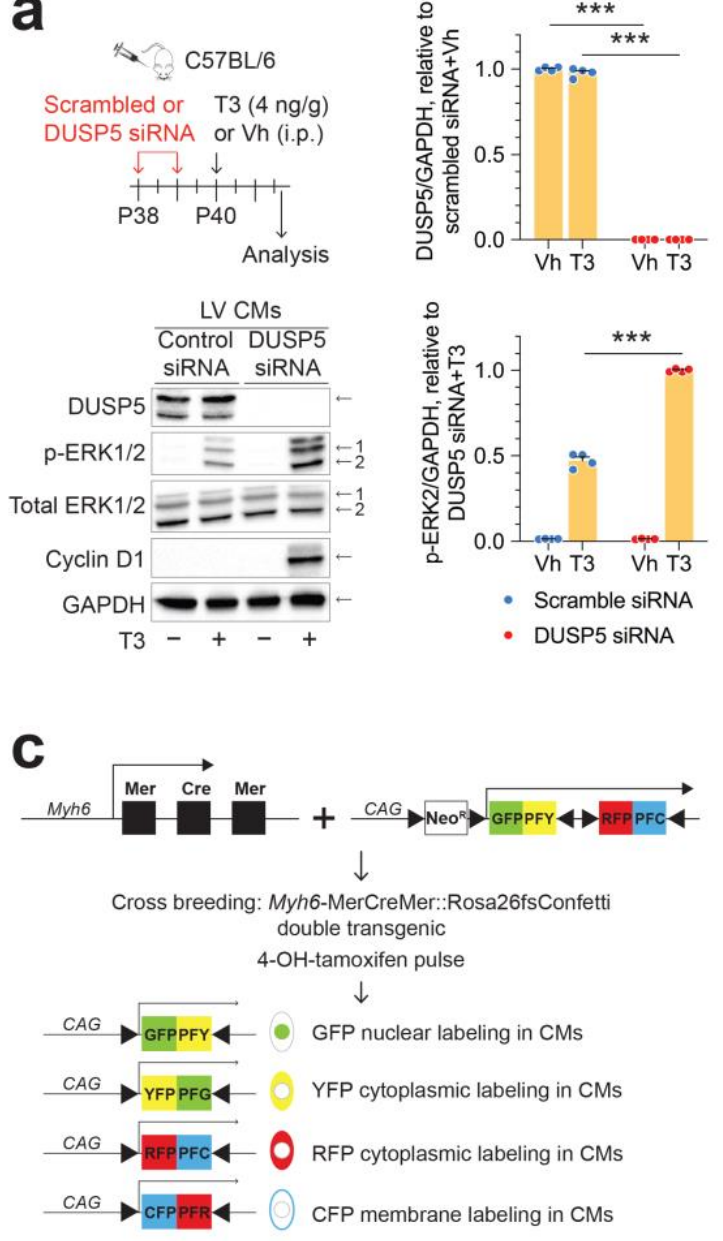

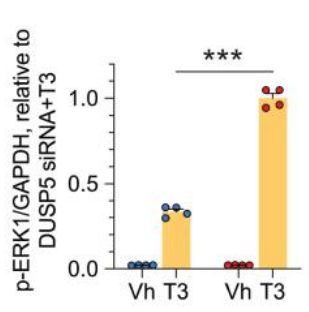
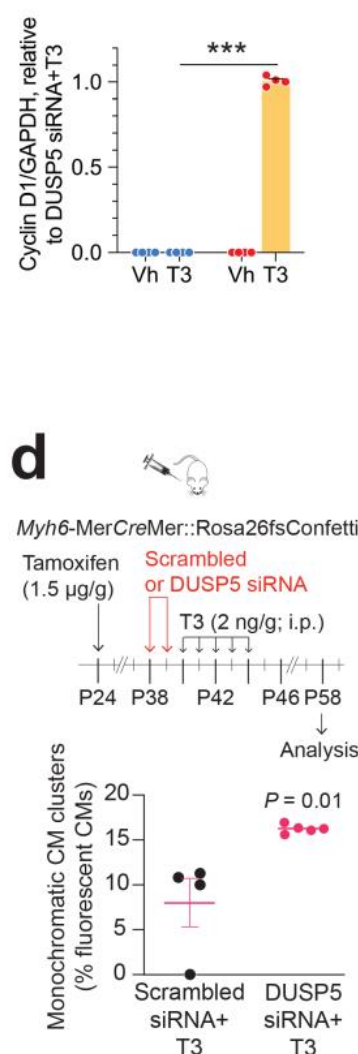

b
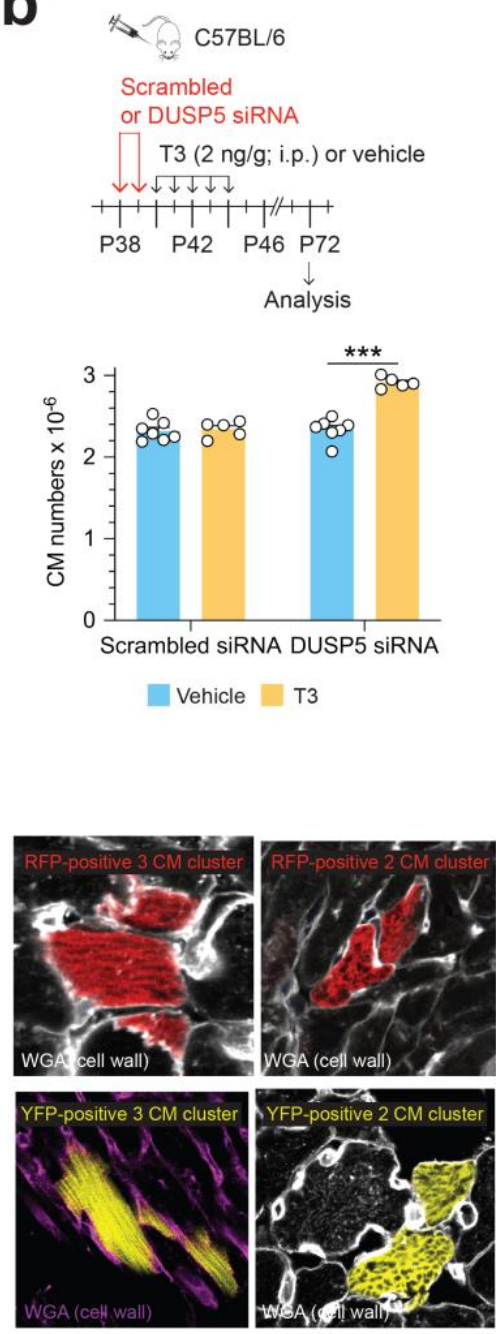

Fig. 1 | DUSP5 siRNA+T3 stimulates p-ERK1/2 accumulation and cardiomyocyte proliferation in young adult mice. a, The experimental protocol (top left) and representative examples of immunoblots (bottom left) and quantitative analysis (right) of p-ERK1/2 and cyclin D1 in LV cardiomyocyte lysates of 5-week-old mice treated with or without DUSP5 siRNA and T3 ( $n=4$ independent biological replicates); uncropped images of immunoblots are available in Extended Data Fig. 7. Vh, vehicle. b, The experimental protocol (top) and ventricular cardiomyocyte numbers (bottom) after in vivo scrambled siRNA, scrambled siRNA+T3, DUSP5 siRNA or DUSP5 siRNA+T3 therapy $(n=5-7$ mice per group); ${ }^{* \star *} P<0.001$. Comparisons were made using ANOVA with Sidak multiple comparison test $(\mathrm{a}, \mathrm{b}) . \mathrm{c}$, The Confetti construct (above) and potential for expression of green (GFP), yellow (YFP), red (RFP) and cyan fluorescent protein (YFP) (below) in cardiomyocytes (below) following Cre-mediated recombination (below). $d$, The experimental protocol (top left), representative examples of monochromatic cardiomyocyte clusters with cell outlines delineated using wheat germ agglutinin (WGA) staining (right) and analysis (bottom left) of DUSP5 siRNA+T3 or scrambled siRNA+T3-driven monochromatic cardiomyocyte cluster formation ( $n=4-5$ mice per group). Mean values \pm s.e.m. Comparison between groups was made using a 2-tailed $t$-test. 
cardiomyocytes labeled with membrane-targeted CFP and nuclear-targeted GFP were infrequent ( $<1$ in 1,000 cells). We avoided the use of higher 4-hydroxytamoxifen doses because it substantially increased replication-independent occurrences of RFP- and YFP-labeled cardiomyocyte cluster as well as the occurrence of bichromatic cardiomyocytes, which complicated lineage tracing.

Two weeks after 4-hydroxytamoxifen treatment, we administered DUSP5 or scrambled siRNA to Myh6-MerCreMer::Rosa26fs-Confetti mice, over 2 days, followed by 5 daily doses of T3. At the time of siRNA treatment, the mice were 5-weeks-old. Hearts were procured 2 weeks later; multiple LV tissue sections from each heart, representing the entire LV, were studied. DUSP5 siRNA+T3 treatment increased the frequency of monochromatic cardiomyocyte clusters by $\sim 2$-fold relative to those found in scrambled siRNA+T3-treated mice $(P=0.01)$ (Fig. 1d). In DUSP5 siRNA+T3 treated Myh6-MerCreMer::Rosa26fs-Confetti mice, monochromatic clusters were found throughout the LV and $\sim 1$ of 6 clusters contained 3 or more cells. However, >2-cell clusters were not found in scrambled siRNA+T3-treated Myh6-MerCreMer::Rosa26fs-Confetti mice. Use of this lineage-tracing tool supports the involvement of multiple individual cardiomyocyte replication events in the LV in response to DUSP5 siRNA+T3 therapy; clonal expansion of a few highly proliferative cardiomyocytes would be evident by the occurrence of cardiomyocyte clusters containing a large number of monochromatic cells14, which we did not observe.

\section{DUSP5 siRNA+T3 therapy builds heart muscle through cardiomyocyte proliferation.}

DUSP5 siRNA+T3 therapy increased LV-to-body weight ratio by $\sim 25 \%$ at 2-weeks post-therapy $(P<0.001)$, without influencing the growth of other heart chambers, of other organs (e.g., the liver, lungs, and the kidneys) or body weights (Table 1). By contrast, neither T3 or DUSP5 siRNA monotherapy altered LV-to-body weight ratios. Thus, administration of DUSP5 siRNA 
Table 1. Body and organ weights and core body temperatures of 5-week-old mice treated with DUSP5 siRNA or scrambled siRNA, with or without T3 therapy

Scrambled siRNAa

\section{Vehicle}

T3
DUSP5 siRNAa

Vehicle T3

\begin{tabular}{|c|c|c|c|c|}
\hline Body weight, $g$ & $21.8 \pm 0.18$ & $21.8 \pm 0.15 \mathrm{~N} . \mathrm{S}$ & $21.8 \pm 0.2$ & $21.7 \pm 0.15$ N.S. \\
\hline Atria/body weight, mg/g & $1.27 \pm 0.015$ & $1.25 \pm 0.015 \mathrm{~N} . \mathrm{S}$ & $1.27 \pm 0.01$ & $1.26 \pm 0.05 \mathrm{~N} . \mathrm{s}$ \\
\hline RV/body weight, mg/g & $1.67 \pm 0.025$ & $1.67 \pm 0.02$ N.S. & $1.67 \pm 0.019$ & $1.60 \pm 0.076 \mathrm{~N} . \mathrm{S}$ \\
\hline LV/body weight, mg/g & $3.31 \pm 0.036$ & $3.3 \pm 0.014$ N.s. & $3.3 \pm 0.027$ & $4.11 \pm 0.04^{\star \star \star}$ \\
\hline Lung/body weight, mg/g & $7.23 \pm 0.036$ & $7.25 \pm 0.012$ N.S. & $7.24 \pm 0.024$ & $7.25 \pm 0.018$ N.S. \\
\hline Kidneys/body weight, mg/g & $21.4 \pm 0.82$ & $21.8 \pm 0.43$ N.S. & $21.6 \pm 0.75$ & $21.7 \pm 0.69$ N.S \\
\hline Liver/body weight, mg/g & $50.5 \pm 0.32$ & $50.5 \pm 0.14$ N.S. & $50.5 \pm 0.37$ & $50.8 \pm 0.36$ N.S. \\
\hline Core body temperature, ${ }^{\circ} \mathrm{C}$ & $37.1 \pm 0.14$ & $37 \pm 0.31$ N.S. & $37 \pm 0.28$ & $37 \pm 0.23 \mathrm{~N} . \mathrm{S}$ \\
\hline$n$ & 6 & 6 & 6 & 6 \\
\hline \multicolumn{5}{|c|}{$\begin{array}{l}\text { aFive-week-old male C57BL/6 mice were given DUSP5 or scrambled siRNA therapy daily for } 2 \text { days. } \\
\text { T3 (2 ng/g, i.p., daily) or vehicle was then administered for } 5 \text { days starting on the day after the } \\
\text { cessation of DUSP5 siRNA therapy. Core body temperatures were assessed at } 2 \text { weeks after cessation } \\
\text { of T3 therapy. Immediately after this measurement, mice were euthanized and body and organ } \\
\text { weights determined. Values are mean } \pm \text { s.e.m. Within group (DUSP5 siRNA-treated or scrambled } \\
\text { siRNA-treated mice) comparisons between vehicle versus T3 treated mice were made using a } 2- \\
\text { tailed t-test. }{ }^{* \star *}<0.001, N . S \text {. (not significant) versus respective vehicle treatments. }\end{array}$} \\
\hline
\end{tabular}

plus T3 selectively increases LV growth.

We used parasternal short-axis M-mode and long-axis B-mode echocardiographic imaging to determine LV dimensions and LV contractile function. Multiple imaging modalities allowed us to assess LV remodeling by determining PW dimensions at the LV mid-papillary and mid-apical level as well as at the LV apex (Extended Data Fig. 2a). These parameters were determined four weeks after administering 2 daily doses of DUSP5 siRNA followed by 5 daily doses of T3 (2 
ng/g, i.p.). Relative to DUSP5 siRNA monotherapy, DUSP5 siRNA+T3 therapy resulted in an increase in PW thickness at the LV apex and the mid-apical level, but not at the mid-papillary level (Extended Data Fig. 2b-d). DUSP5 siRNA+T3-driven remodeling also resulted in a longer and narrower LV cavity, based on measurements of minor and major axis LV internal dimensions at diastole (LVID-d) (Extended Data Fig. 2e-h). Consistent with these changes, LV mass increased by $\sim 10 \%(P<0.001)$ (Extended Data Fig. 2a-f). Of note, however, the formula used by the Vevo software employed here to calculate LV mass using M-mode imaging [1.05 × ((LVID-d + LVPW-d + IVS-d) 3 - LVID-d3) mg] assumes constant LV wall thickness (based on measurements at the mid-papillary level). Because of uneven changes in LV PW after DUSP5 siRNA+T3 therapy the LV mass measurement is an underestimate; thus, we found that DUSP5 siRNA+T3 therapy increased LV mass by $\sim 10 \%$, but LV-to-body weight ratio increased by $\sim 25 \%$. In contrast to the effects of DUSP5 siRNA+T3 therapy, T3 had no discernable effects when combined with scrambled siRNA therapy (Extended Data Fig. 2a-f). Sequential temporal analysis showed that LV mass gradually increases after cessation of DUSP5 siRNA+T3 therapy, with major changes occurring between 1 and 2 weeks after therapy (Extended Data Fig. 3a-f).

Evaluations of LV volumes at end-diastole (EDV) and end-systole (ESV), four weeks after DUSP5 siRNA+T3 therapy showed an 14-percentage point increase in ejection fraction [(EDV - ESV) $\times 100 / E D V](P<0.0001)($ Extended Data Fig. 4a). In keeping with this increase, LV fractional shortening increased $\sim 45 \%(P<0.0001)$ (Extended Data Fig. 4b). DUSP5 siRNA+T3 therapy decreased EDV by $\sim 30 \%(P<0.0001)$ (Extended Data Fig. 4c). Because of an even greater decrease in ESV (by 60\%; $P<0.0001$ ) (Extended Data Fig. 4d), stroke volume (EDV ESV) was not significantly changed (Extended Data Fig. 4e). By contrast, scrambled siRNA therapy, with or without T3, was without effect (Extended Data Fig. 4a-e). Representative videos showing LV trace of parasternal long-axis B-mode images of mouse hearts between full 
diastole and full systole after therapy with scrambled siRNA+T3 (Extended Data Video 1) or DUSP5 siRNA+T3 (Extended Data Video 2) are provided. Thus, collectively, our data show that DUSP5 siRNA+T3 therapy induces cardiomyocyte proliferation, which results in a LV PW that is more muscular and has enhanced contractility.

To determine if cardiomyocyte proliferation is central to sustained changes in LV dimensions and contractile functional, we studied the effect of danusertib - a potent, small molecule inhibitor of aurora kinases that blocks the cell cycle by interfering with the normal progression of mitosis15- on DUSP5 siRNA+T3-driven changes in LV remodeling and contractile function. Danusertib (15 mg/kg, i.p., b.i.d) or its vehicle (50\% DMSO:50\% PBS) were administered to mice at the start of DUSP5 siRNA treatment (for 2 consecutive days) and then continued for a total of 7 days. Immediately after the 2-day of DUSP5 siRNA treatment, mice received T3 or vehicle therapy for 5 days, as above. LV dimensions and function were assessed 2 weeks after the end of therapy and cardiomyocyte numbers were determined 24-48 hours later (Fig. 2a). Danusertib not only inhibited DUSP5 siRNA+T3-induced increases in cardiomyocyte numbers (Fig. 2b), LV mass (Fig. 2c) and LV PW thickness (Fig. 2d,e), but also increases in LV ejection fraction (Fig. 2f) and fractional shortening (Fig. 2g). These data suggest that DUSP5 siRNA+T3driven expansion of the LV myocardium and improvements in its contractile function require cardiomyocyte proliferation. 


\section{a}

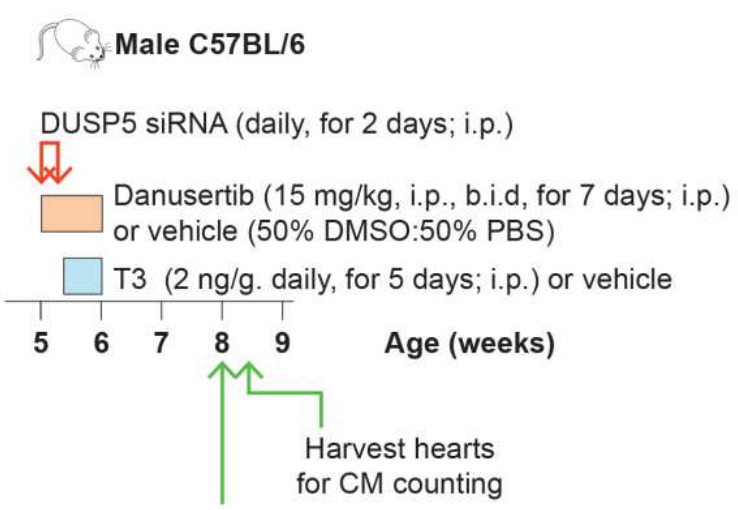

Echocardiography
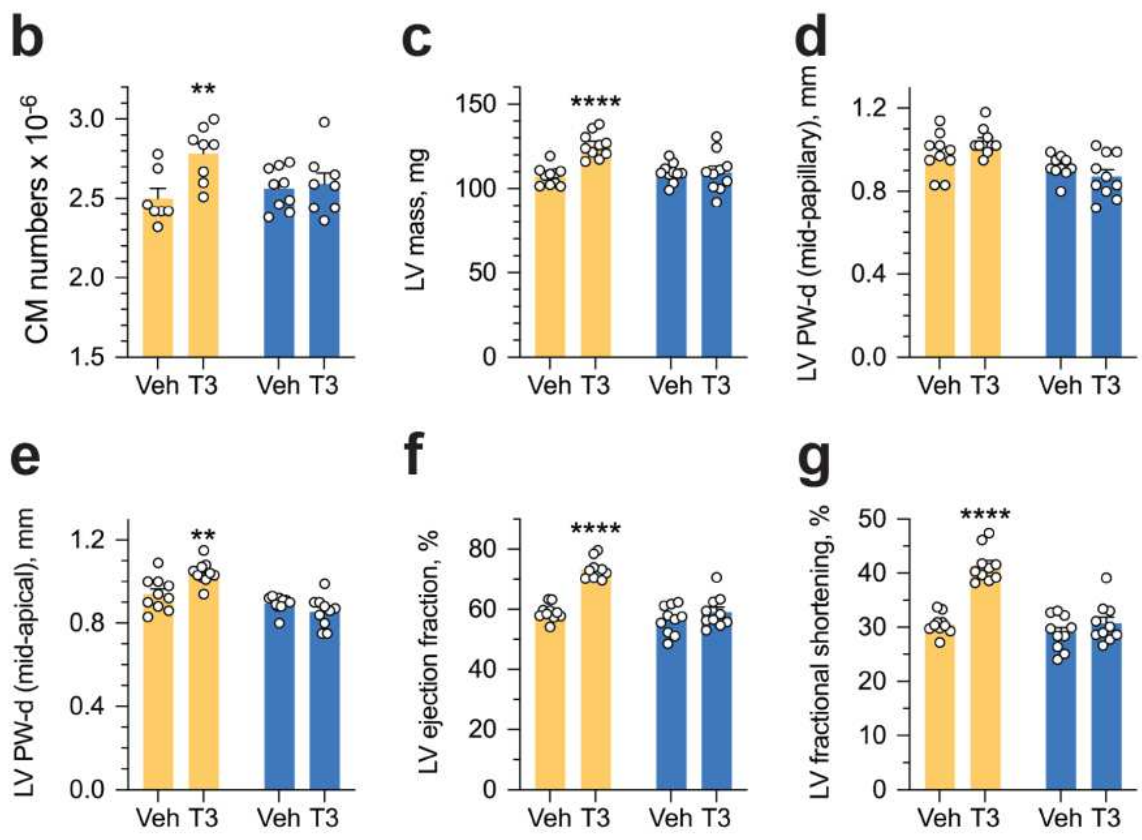

50\% DMSO:50\% PBS+DUSP5 siRNA

Danusertib+DUSP5 siRNA

Fig. 2 | Cardiomyocyte proliferation drives LV growth and increases in LV contractile function after DUSP5 siRNA+T3 therapy. a, Experimental protocol for testing the effects of the cell cycle inhibitor, danusertib, on T3-stimulated increases in LV dimensions and contractile function in DUSP5 siRNA-treated mice. b, T3-stimulated increase in ventricular cardiomyocyte numbers in DUSP5 siRNA+T3 treated mice are blocked by danusertib ( $n=6-8$ mice per group); ${ }^{\star} *<<0.01 . c-g$, In vivo echocardiographic evaluation of T3-stimulated changes in LV mass (c), LV PW dimensions, at diastole, at the LV mid-papillary level (d) and at the LV apex (e), LV ejection fraction ( $f$ ) and LV fractional shortening $(\mathrm{g})$ in DUSP5 siRNA+T3 treated mice, with or without danusertib treatment $(n=10$ mice per group); ${ }^{*} P<0.01,{ }^{\star} * \star \star * P<0.0001$. Mean values \pm s.e.m. Comparisons were made using ANOVA with Sidak multiple comparison test; only within group P-values are shown $(b-g)$. 
Repeated doxorubicin exposure induces cardiomyopathy. Given that girls are at greater risk of developing LV dysfunction after anthracycline therapy than boys16, we studied the effects of repeated doxorubicin exposure on the LV of young female mice. Five-week-old female mice were given 3 doses of doxorubicin ( $20 \mu \mathrm{g} / \mathrm{g}$, i.p.) at 2-weekly intervals, starting at 5-weeks-ofage. Echocardiography was performed 5 days after the last dose to assess cardiotoxicity. The experimental protocol is illustrated in Fig. 3a. We compared the LVs of doxorubicin-injured hearts with untreated age-matched controls. Repeated doxorubicin exposure resulted in a $45 \%$ increase in EDV (Fig. 3b) and a 3.6-fold increase in ESV (Fig. 3c) $(P<0.001$, in each case). These $\mathrm{LV}$ volume changes produced a $\sim 40$-percentage point decrease in ejection fraction $(P<$ 0.0001) (Fig. 3d) and a 32\% decrease in stroke volume $(P<0.0001)$ (Fig. 3e). Associated with LV dysfunction, LV mass decreased by $32 \%(P<0.0001)$ (Fig. 4a) and PW thicknesses at the LV mid-papillary and mid-apical level and the LV apex decreased between 25\%-30\% ( $P<$ 0.001 in each case) (Fig. 4b). Thus, the main effects of repeated doxorubicin exposure were: loss of LV muscle, as assessed by decreased LV mass and global LV PW thinning; LV dilatation, evident by increased EDV; and marked LV dysfunction that was evident from an increase in ESV and decreases in ejection fraction and stroke volume.

\section{DUSP5 siRNA+T3 therapy rebuilds the myocardium of mice with doxorubicin}

cardiomyopathy. Restoration of LV muscle and associated improvement in LV contractile performance are important goals of regenerative therapy. We examined the impact of combination therapy with DUSP5 siRNA and T3 in mice with chronic doxorubicin cardiomyopathy (see above). In addition to DUSP5 siRNA+T3 therapy, which stimulated cardiomyocyte proliferation (Fig. 1b), we studied the effects of scrambled siRNA therapy, which did not (Fig. 1b). Post-therapy effects were studied over 3 weeks (Fig. 3a). An untreated mouse 
a

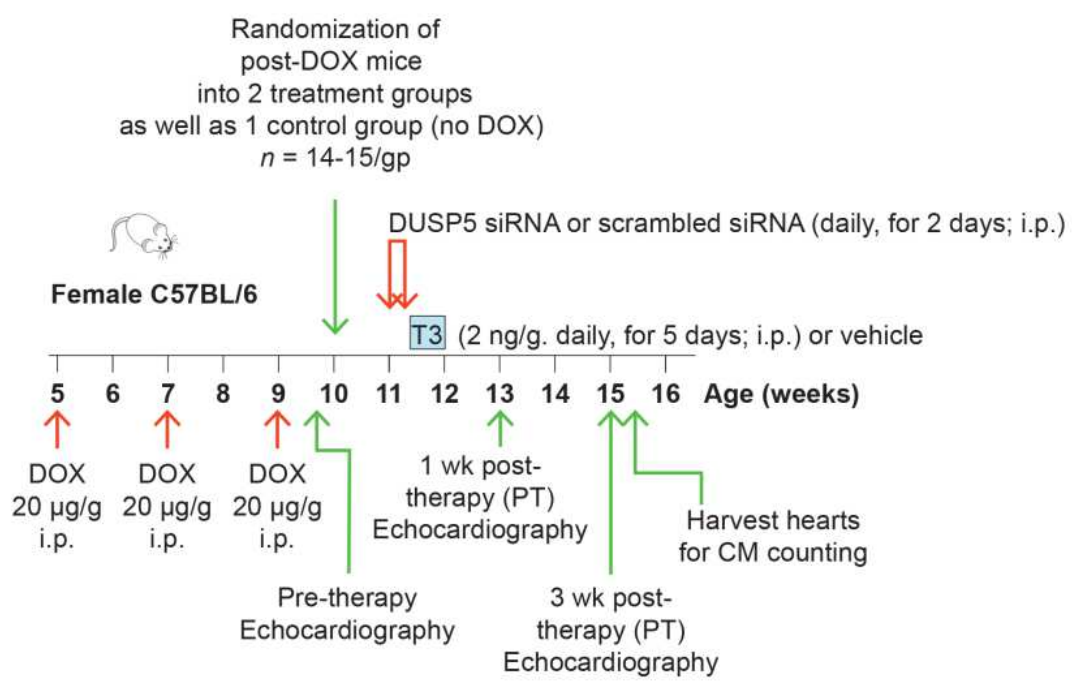

b

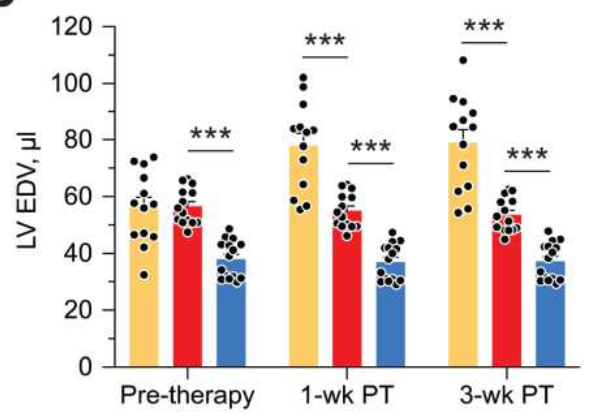

d

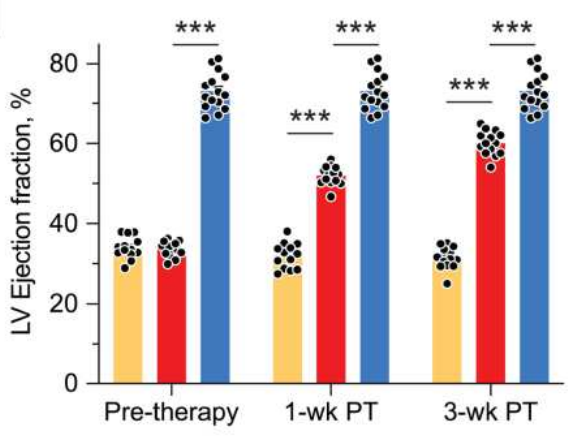

C

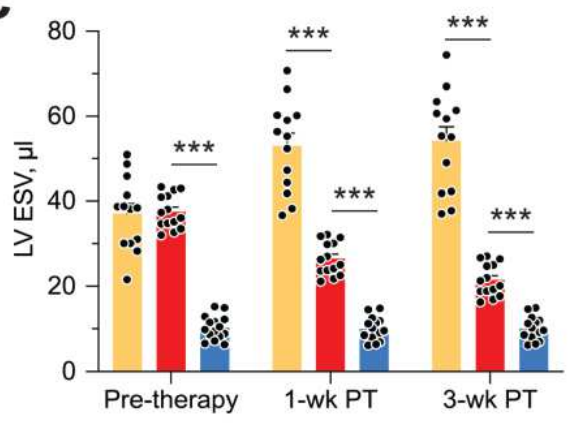

e

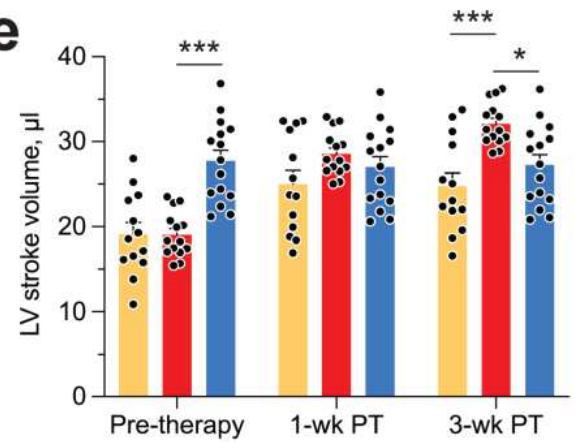

\section{DOX+Scrambled siRNA DOX+DUSP5 siRNA+T3 age-matched controls}

Fig. 3 | Transient DUSP5 siRNA+T3 therapy reverses LV dysfunction in doxorubicin-injured hearts. a, Diagram showing the experimental protocol used to create doxorubicin-induced cardiotoxicity and implementation of transient therapies with scrambled siRNA (control) or DUSP5 siRNA+T3 therapy. be, Repeated assessments in mice of LV end-diastolic volume (EDV) (b), end-systolic volume (ESV) (c), LV ejection fraction (d) and LV stroke volume (e) at the end of repeated doxorubicin exposure (pretherapy) and 1- and 3-week after scrambled siRNA or DUSP5 siRNA+T3 therapy $(n=13-14$ mice/group). These temporal assessments were also made in age-matched controls that were not exposed to doxorubicin or any therapy $(n=15)$. ${ }^{* \star *} P<0.0001$. Data are represented as mean values \pm s.e.m. Comparisons with the doxorubicin+DUSP5 siRNA+T3 group were made using ANOVA with Sidak multiple comparison test. PT, post-therapy; DOX, doxorubicin; CM, cardiomyocyte. 

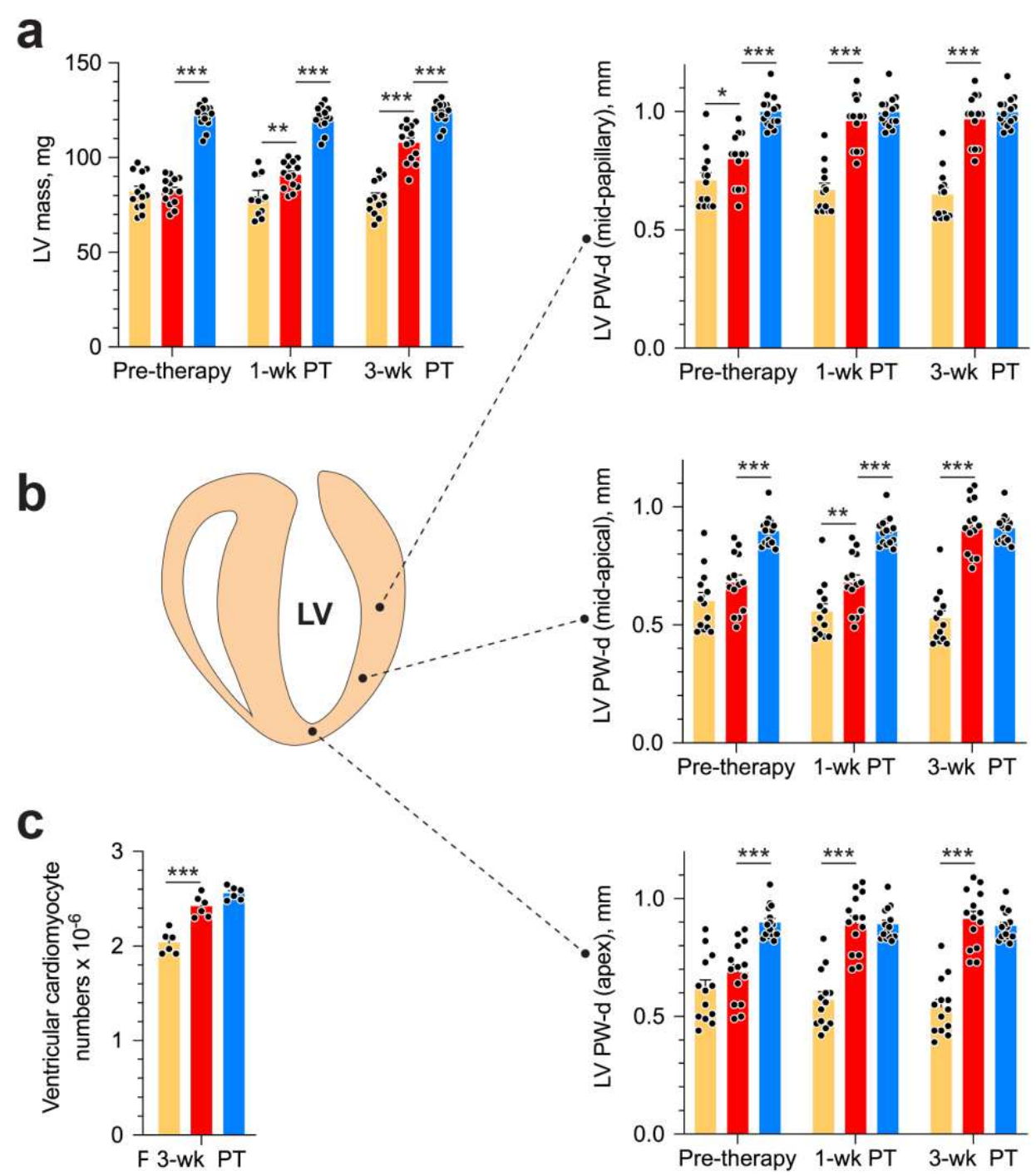

DOX+Scrambled siRNA

DOX+DUSP5 siRNA+T3

Age-matched controls

Fig. 4 | Transient DUSP5 siRNA+T3 therapy rebuilds cardiac muscle in mice with doxorubicin cardiomyopathy. a,b, Repeated assessments in mice of LV mass (a) and posterior wall (PW) dimensions at end-diastole (b) at the LV mid-papillary level (top), mid-apical level (middle) and at the LV apex (bottom); assessments were made at the end of repeated doxorubicin exposure (pretherapy) and 1- and 3-week after scrambled siRNA (control therapy) or DUSP5 siRNA+T3 therapy ( $n=$ 13-14 mice/group). Echocardiographic sampling sites are indicated in the diagram (middle left). Agematched controls that were not exposed to doxorubicin or any therapy were also studied $(n=15)$. ${ }^{* *} P$ $<0.01$, ${ }^{* \star *} P<0.001$. Mean values \pm s.e.m. Comparisons with the doxorubicin+DUSP5 siRNA+T3 group were made using ANOVA with Sidak multiple comparison test. PT, post-therapy; DOX, doxorubicin. c, Total ventricular cardiomyocyte numbers in doxorubicin-treated mice were determined 3-weeks after scrambled siRNA or DUSP5 siRNA+T3 therapy as well as in untreated agematched controls ( $n=6-7$ mice per group). Mean values \pm s.e.m. ${ }^{\star * \star} P<0.00$. Comparisons with the doxorubicin+DUSP5 siRNA+T3 group were made using ANOVA with Sidak multiple comparison test. 
group in which mice were neither exposed to doxorubicin nor therapy served as age-matched controls.

We first assessed the effect of DUSP5 siRNA+T3 therapy on ventricular cardiomyocyte numbers. Doxorubicin-treated mice given DUSP5 siRNA+T3 therapy had 19\% more cardiac muscle cells in their ventricles than those given scrambled siRNA therapy $(P<0.001)$ (Fig. 4c). In these DUSP5 siRNA+T3-treated mice, ventricular cardiomyocyte numbers were similar to those of age-matched controls $(P=0.068)$ (Fig. $4 \mathrm{c})$. LV mass increased by $31 \%$ after DUSP5 siRNA+T3 therapy $(P<0.0001)$, but not after scrambled siRNA therapy $(P=0.8)$ (Fig. $4 a)$. These results raise the possibility that DUSP5 siRNA+T3 therapy regenerates the LV muscle lost as a result of repeated doxorubicin exposure.

In uninjured hearts, the increase in LV PW observed with DUSP5 siRNA+T3 therapy was not uniform, with major effects seen in the periapical LV myocardium (Extended Data Fig. 2bd). But LV PW thinning after repeated doxorubicin exposure is global (Fig. 4b). To determine if similar non-uniformity of LV muscle regeneration is also observed with DUSP5 siRNA+T3 treatment of doxorubicin-injured hearts, we determined PW thickness at multiple LV levels. This showed that after DUSP5 siRNA+T3 therapy, PW thickness was similar to that of age-matched controls at all levels throughout the LV, that is, at the LV mid-papillary and mid-apical levels and at the LV apex, whereas before the start of therapy they were all about 20\%-33\% thinner, respectively $(P<0.001)$ (Fig. 4b). No such LV PW thickening was observed after scrambled siRNA therapy (Fig. 4b). Gross appearance and histology of the heart after DUSP5 siRNA+T3 or scrambled siRNA therapy are shown in Fig. 5a. and 5b, respectively. In the rebuilt LV myocardium of DUSP5 siRNA+T3-treated mice, cardiomyogenesis was apparent by the finding that LV PW cardiomyocyte and capillary densities were $48 \%$ and $57 \%$ higher, respectively, than in mice treated with scrambled siRNA ( $P<0.0001$ in both cases) (Fig. $5 \mathrm{c}$, d), while the capillary- 

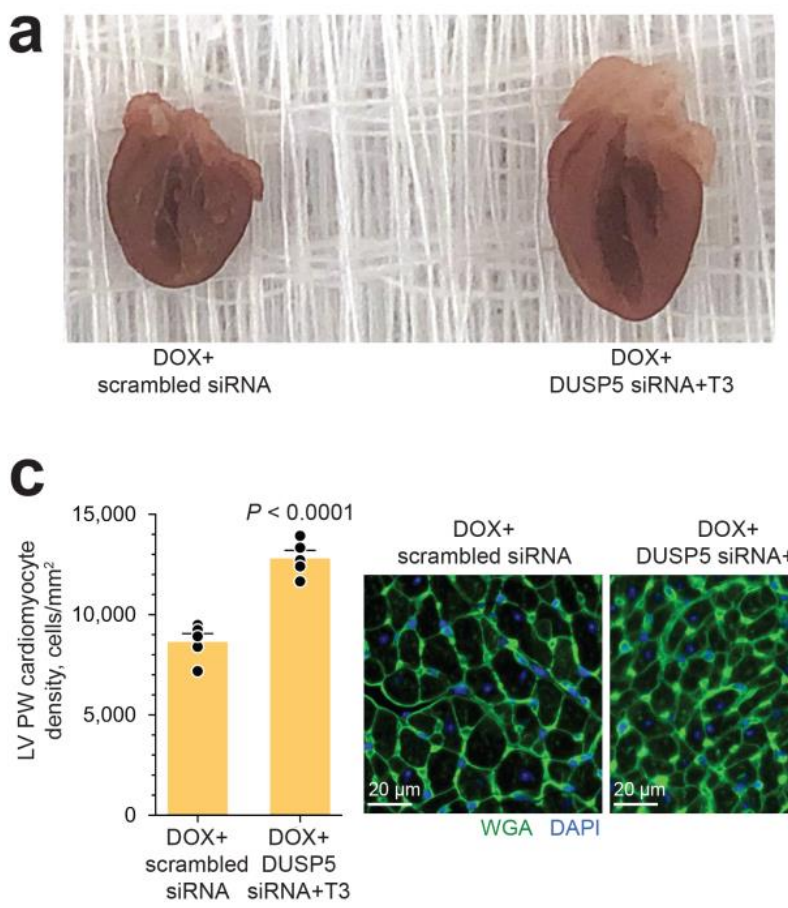
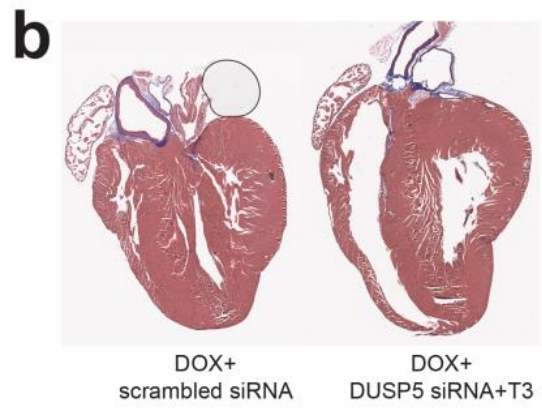

d
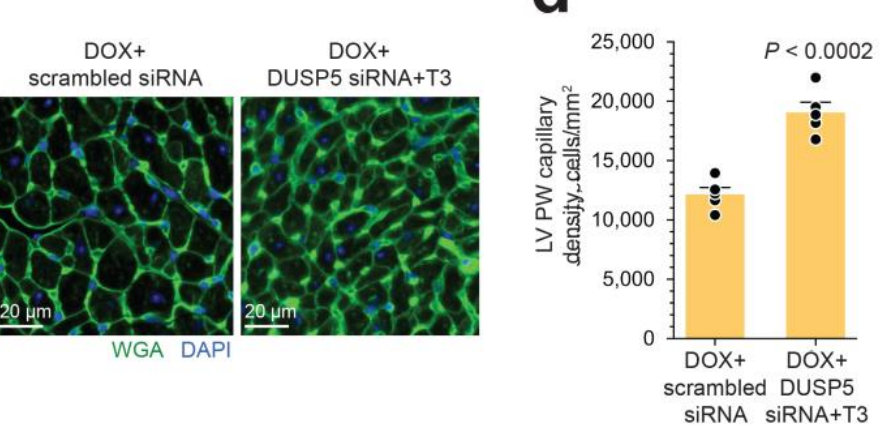

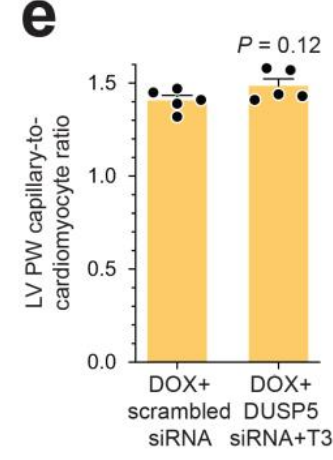

Fig. 5 | Gross morphology and cellular composition of doxorubicin-injured LVs following DUSP5 siRNA+T3 therapy. a,b, Gross appearance of mouse hearts cut in the longitudinal plane (a) and trichrome-stained tissue sections (b) of doxorubicin-injured hearts studied at 3-weeks following DUSP5 siRNA+T3 or control scrambled siRNA therapy. c,d, LV PW cardiomyocyte (c) and capillary densities (d) were assessed after staining with wheat-germ agglutinin (WGA) and 4, 6-diamidino-2phenylindole (DAPI) to identify cell borders and nuclei, respectively. Representative images are shown in c (right). Bars, $20 \mu \mathrm{m}$. Determinations were made using tissue sections from the LV mid-papillary region. e, Capillary-to cardiomyocyte ratios. Comparison between groups were made using a 2-tailed $t$-test $(n=5$ mice per group in c-e). Data are represented as mean values \pm s.e.m.

to-cardiomyocyte ratio was maintained at $\sim 1.4$ (Fig. $5 \mathrm{e}$ ).

To evaluate the impact of regenerative therapy on the ability of the LV PW to contract, we compared LV PW dimensions at systole and diastole, before and then 3 weeks after DUSP5 siRNA+T3 therapy. LV PW dimensions (assessed at the mid-papillary level) at end-diastole and end-systole increased by $22 \%$ and $30 \%(P<0.001$, in each case), respectively, after regenerative therapy (Extended Data Fig. 5). As a result, systole-associated absolute LV PW thickening (LV PW-s - LV PW-d) (Extended Data Fig. 5), which is a measure of localized LV PW contractility, increased by $54 \%(P=0.014)$. Global LV contractility also increased, as evidenced by a $26-$ 
percentage point increase in LV ejection fraction $(P<0.0001)$ and a $68 \%$ increase in stroke volume $(P<0.0001)$ (Fig. 3d,e).

We next asked if these therapy-induced changes in LV PW are sufficient to reverse the contractile deficit resulting from repeated doxorubicin exposure? Before the start of DUSP5 siRNA+T3 therapy, LV ejection fraction was $~ 40$-percentage point lower than that of agematched controls (Fig. 3d) and stroke volume was 32\% lower (Fig. 3e) $(P<0.0001$, in each case), whereas 3 -weeks after therapy, they were $\sim 13$-percentage point lower and $12 \%$ higher than age-matched controls, respectively $(P<0.0001$, in each case) (Fig. 3d,e), indicating substantial restoration of LV contractile function.

We also investigated the effect of therapy on doxorubicin-induced LV dilation. By the end of repeated doxorubicin treatments, LV chambers were about 1.45 -fold larger than those of agematched controls $(P<0.0001)$ (Fig. 3b). Following scrambled siRNA therapy, they continued to enlarge, such that by 3 -weeks post-therapy they were 2.1 -fold larger $(P<0.0001)$ (Fig. 3b). Extant LV dilatation was not reversed by DUSP5 siRNA+T3 therapy, but it completely prevented the progressive chamber dilatation, evident from increases in EDVs, that was observed in animals treated with scrambled siRNA (Fig. 3b).

Long-term doxorubicin-treated mice given acute DUSP5 siRNA+T3 therapy generally looked well: they were not lethargic, had normal posture and all 14 mice survived over the 3week post-therapy follow-up. By comparison, doxorubicin-treated mice given scrambled siRNA therapy were in poor health. All 14 animals were lethargic or immobile, had a hunched posture, were poorly groomed (Extended Data Fig. 6) and one died in the post-therapy period. In addition, 3-weeks post-therapy, doxorubicin-treated mice given scrambled siRNA therapy had lower body weights than those given DUSP5 siRNA+T3 therapy (body weights were $16.6 \pm 0.63$ $\mathrm{g}$ and $18.5 \pm 0.45 \mathrm{~g}$ for mice given scrambled siRNA and DUSP5 siRNA+T3 therapy, respectively; $P<0.05)$. Because our predetermined criteria for signs of pain included lack of 
grooming, sitting in a hunched position, abdominal breathing and body weight loss, we had to euthanize the mice at 3-weeks post therapy and terminate the study.

\section{Discussion}

The evidence presented here suggests that brief (7-day) therapy with DUSP5 siRNA+T3 builds LV muscle in uninjured hearts and rebuilds LV muscle in chronic doxorubicin-injured hearts. In uninjured hearts, DUSP5 siRNA+T3 therapy increased cardiomyocyte endowment of the heart, enhancing LV thickness and contractile function; effects that were sustained for up to 4-weeks post-therapy. Danusertib, an inhibitor of mitosis15, prevented these salutary effects of DUSP5 siRNA+T3 therapy, which suggests that an expanded LV myocardium with improved contractile function requires cardiomyocyte proliferation. Thus, short term DUSP5 siRNA+T3 treatment is a viable therapeutic approach to treating doxorubicin cardiomyopathy, a condition for which no specific therapy is currently available17. Moreover, as an acute pharmacological approach it carries the additional benefit of potentially being rapidly translatable into an effective therapeutic strategy for the management of patients with doxorubicin cardiomyopathy.

We demonstrate that transient therapy with DUSP5 siRNA+T3 therapy rebuilds heart muscle in $~ 12$-week-old adult mice with chronic doxorubicin cardiomyopathy through cardiomyocyte proliferation (Figs. 4c and 5c) and neovascularization (Fig. 5d); processes that, within a few weeks after the therapy, normalize the anatomy (Fig. 5a) and contractile function (Fig. 3c-e and Extended Data Fig. 5a-c) of the injured LV. Taken together, our data show that the limited regenerative potential of adult hearts can be therapeutically enhanced.

Although DUSP5 siRNA+T3 therapy did not regress pre-therapy LV chamber enlargement, it completely prevented the continued marked LV dilatation observed in doxorubicin-treated animals given scrambled siRNA control therapy. This contrasts with present therapies for doxorubicin and other forms of dilated cardiomyopathy, which do not ameliorate progressive LV 
chamber enlargement18, although it will be of interest to see, in future studies, if combining these agents with combined DUSP5 siRNA plus T3 therapy, provides even greater reversal of adverse LV remodeling.

ERK1/2 phosphorylation and translocation of the phosphoprotein from the cytosol to the nucleus connects growth factor signaling with transcriptional activation of cell cycle promoting genes and cell proliferation19,20. In neonatal P2 cardiomyocytes, ERK1/2 activation is required for T3-stimulated cardiomyocyte proliferation10. As LV cardiomyocytes mature, the expression of DUSP5, a nuclear phosphatase that inhibits p-ERK1/2, increases, starting immediately after the neonatal period (which ends at P6)11. We show here that, in young adult mice, DUSP5 knockdown in LV cardiomyocytes using DUSP5 siRNA amplifies T3-stimulated increases in pERK1/2 and cyclin D1 and promotes cardiomyocyte proliferation.

To establish the extent of cardiomyocyte proliferation, we used direct cell counting after enzymatic heart disaggregation. Determination of the percentage of cardiomyocytes that are in mitosis (e.g., using phospho-histone 3-labeling), in comparison, provides information about cell cycle activity at the moment of tissue procurement, but not the cumulative effect of therapy or the number of cells that ultimately undergo cytokinesis. While lineage tracing tools are valuable in defining replicative strategies used by cells (e.g., clonal expansion14) they are not designed to estimate the number of cardiomyocytes that are added as a result of therapy. For these reasons, direct cell counting is widely used to determine the extent of cardiomyocyte proliferation8,21-23; it also avoids problems associated with indirect methods24,25, such as cell shrinkage, sampling area and cell boundary determination26. Incomplete tissue digestion is a limitation of the direct cell counting method 26 . Here we have used an optimized enzymatic heart disaggregation protocolio to achieve digestion efficiencies that exceed $97 \%$.

T3 effects are dependent on dose and duration of therapy. At high doses, exogenous T3 administration (e.g., 15-50 ng/g), when sustained over long periods (e.g., for 21 days), results in 
hyperthermia, body weight loss and increased heart weight 27 . We administered T3, $2 \mathrm{ng} / \mathrm{g} / \mathrm{day}$, over 5 days as a component of the cardiac regeneration protocol. In the absence of DUSP5 siRNA treatment, this dose/duration of T3 therapy did not have an enduring impact on heart or body weight and did not increase body temperature. Also, we found no evidence of cardiac arrhythmias in dozens of echocardiographic analyses performed on T3-treated mice with or without DUSP5 siRNA therapy.

A key question is how intraperitoneal administration of the DUSP5 siRNA molecule can result in target specificity. T3 activates ERK1/2 proliferative signaling in cardiomyocytes secondary to increased IgfI and IgfIr expression, both of which are required for this T3 action 10. Although Igfl has a validated thyroid responsive element (TRE) in its promoter28, T3 stimulates Igfl transcription in cardiomyocytes through a redox mechanism that activates a rare c-Jun Nterminal kinase (JNK) isoform, JNK2 $\alpha 2$ 10. The molecular mechanism by which T3 activates IgfIr transcription in cardiomyocytes is uncertain. Molecular heterogeneity in signaling mechanisms between cells is likely to dictate which cells proliferate in response to T3. An examination of organ and body weights revealed that low-dose T3, in combination with DUSP5 siRNA therapy, selectively increases LV growth, but not that of other heart chambers or of many other organs examined in our study.

Polyethylenimine-derived in vivo-jetPEI $\circledast$ transfection reagent was used to deliver siRNA into the mouse; it is also an effective means of delivering DNA and RNA in humans29,30, which potentially makes DUSP5 siRNA+T3 therapy easily translatable. Doxorubicin is one of the most important antineoplastic drugs but, repeated use, as in cancer therapy, amplifies its cardiotoxicity, thereby limiting its antitumor efficacy 18. Given the findings here, transient DUSP5 siRNA+T3 therapy may prove useful for sustained restoration of LV muscle and contractile function in patients with doxorubicin cardiomyopathy. 


\section{Online content}

Methods, additional references, Nature Research reporting summaries, source data, extended data, supplementary information, acknowledgements, peer review information, details of author contributions and competing interests, and statements of data and code availability.

\section{References}

1. van Dalen, E. C., Raphaël, M. F., Caron, H. N. \& Kremer L. C. M. Treatment including anthracyclines versus treatment not including anthracyclines for childhood cancer. Cochrane Database Syst. Rev. 9, CD006647 (2014).

2. Oeffinger, K. C. et al. Chronic health conditions in adult survivors of childhood cancer. $N$. Engl. J. Med. 355, 1572-1582 (2006).

3. McGowan, J. V. et al. Anthracycline chemotherapy and cardiotoxicity. Cardiovasc. Drugs Ther. 31, 63-75 (2017).

4. Zhang, S. et al. Identification of the molecular basis of doxorubicin-induced cardiotoxicity. Nat. Med. 18, 1639-1642 (2012).

5. Simões R. et al. Troponin as a cardiotoxicity marker in breast cancer patients receiving anthracycline-based chemotherapy: A narrative review. Biomed. Pharmacother. 107, 989996 (2018).

6. Hassink, R. J. et al. Cardiomyocyte cell cycle activation improves cardiac function after myocardial infarction. Cardiovasc. Res. 78, 18-25 (2008).

7. Shapiro, S. D. et al. Cyclin A2 induces cardiac regeneration after myocardial infarction 
through cytokinesis of adult cardiomyocytes. Sci. Transl. Med. 6, 224ra27 (2014).

8. D'Uva, G. et al. ERBB2 triggers mammalian heart regeneration by promoting cardiomyocyte dedifferentiation and proliferation. Nat. Cell Biol. 17, 627-638 (2015).

9. Nakada, Y. et al. Hypoxia induces heart regeneration in adult mice. Nature 541, 222-227 (2017).

10. Tan, L. et al. Redox activation of JNK2 22 mediates thyroid hormone-stimulated proliferation of neonatal murine cardiomyocytes. Sci. Rep. 9, 17731 (2019).

11. Bogush, N. et al. DUSP5 expression in left ventricular cardiomyocytes of young hearts regulates thyroid hormone (T3)-induced proliferative ERK1/2 signaling. (in review). A copy of this manuscript is supplied as "Additional Review Material."

12. Mebratu, Y. \& Tesfaigzi, Y. How ERK1/2 activation controls cell proliferation and cell death: is subcellular localization the answer? Cell Cycle 8, 1168-1175 (2009).

13. Snippert, H. J. et al. Intestinal crypt homeostasis results from neutral competition between symmetrically dividing Lgr5 stem cells. Cell 143, 134-144 (2010).

14. Gupta, V. \& Poss, K. D. Clonally dominant cardiomyocytes direct heart morphogenesis. Nature 484, 479-484 (2012).

15. Meulenbeld, H. J., Mathijssen, R. H., Verweij, J., de Wit, R. \& de Jonge, M. J. Danusertib, an aurora kinase inhibitor. Expert Opin. Investig. Drugs 21, 383-393 (2012).

16. Silber, J. H., Jakacki, R. I., Larsen, R. L., Goldwein, J. W. \& Barber, G. Increased risk of cardiac dysfunction after anthracyclines in girls. Med. Pediatr. Oncol. 21, 477-479 (1993).

17. Takemura, G. \& Fujiwara, H. Doxorubicin-induced cardiomyopathy from the cardiotoxic mechanisms to management. Prog. Cardiovasc. Dis. 49, 330-352 (2007). 
18. Mukku, R. B. et al. Heart failure therapies for end-stage chemotherapy-induced cardiomyopathy. J. Card. Fail. 22, 439-448 (2016).

19. Brunet, A. et al. Nuclear translocation of p42/p44 mitogen-activated protein kinase is required for growth factor-induced gene expression and cell cycle entry. EMBO J. 18, 664674 (1999).

20. Yamamoto, T. et al. Continuous ERK activation downregulates antiproliferative genes throughout G1 phase to allow cell cycle progression. Curr. Biol. 16, 1171-1182 (2006).

21. Puente, B.N. et al. The oxygen-rich postnatal environment induces cardiomyocyte cell-cycle arrest through DNA damage response. Cell 157, 565-579 (2014).

22. Naqvi, et al. A proliferative burst during preadolescence establishes the final cardiomyocyte number. Cell 157, 795-807 (2014).

23. Hirose, K. et al. Evidence for hormonal control of heart regenerative capacity during endothermy acquisition. Science 364, 184-188 (2019).

24. Chaudhry, H. W. et al. Cyclin A2 mediates cardiomyocyte mitosis in the postmitotic myocardium. J. Biol. Chem. 279, 35858-35866 (2004).

25. Alkass, K. et al. No evidence for cardiomyocyte number expansion in preadolescent mice. Cell 163, 1026-1036 (2015).

26. Foglia, M. J. \& Poss, K. D. Building and re-building the heart by cardiomyocyte proliferation. Development 143, 729-740 (2016).

27. Bürgi, U. et al. Food intake, body and heart composition, and heart rate in T3 plus atenololtreated rats. Am. J. Physiol. 256, E459-E466 (1989).

28. Xing, W. et al. Genetic evidence that thyroid hormone is indispensable for prepubertal insulin-like growth factor-I expression and bone acquisition in mice. J. Bone Miner. Res. 27, 
1067-1079 (2012).

29. Poeck, H. et al., 5'-triphosphate-siRNA: turning gene silencing and Rig-I activation against melanoma. Nat. Med. 14, 1256-1263 (2008).

30. Lisziewicz, J. et al. Single DermaVir immunization: dose-dependent expansion of precursor/memory T cells against all HIV antigens in HIV-1 infected individuals. PLoS One 7, e35416 (2012). 


\section{Figures}

a
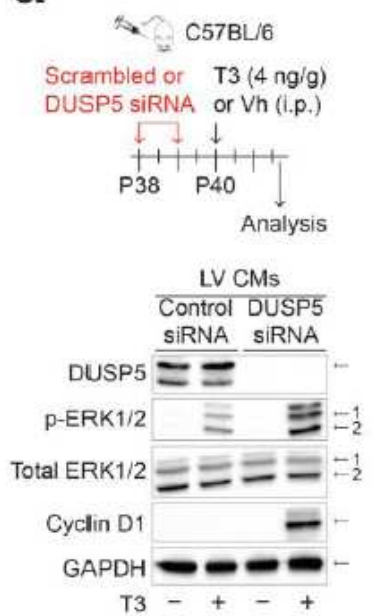

T3
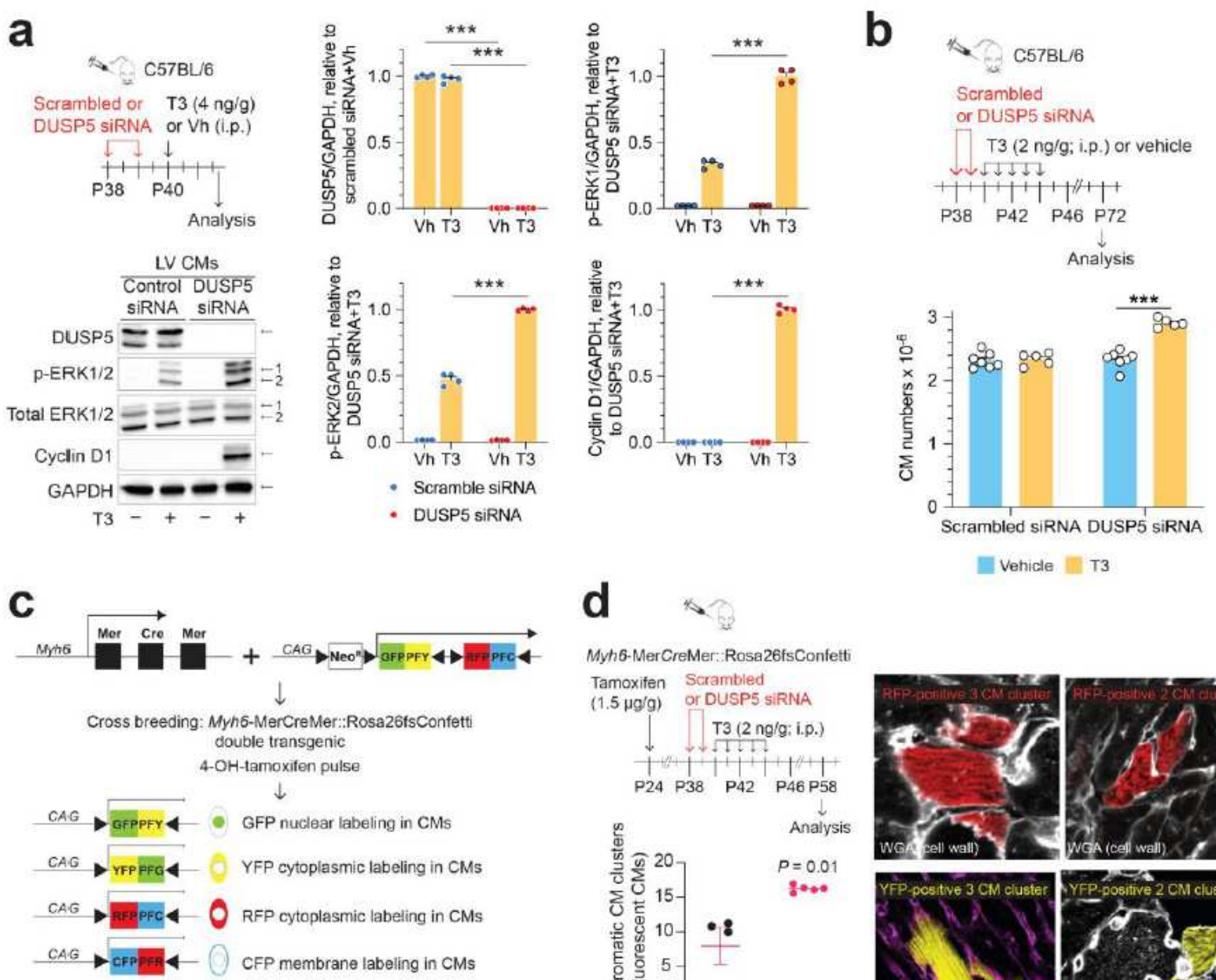
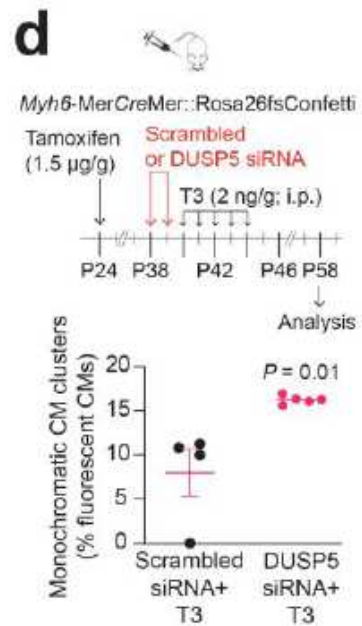
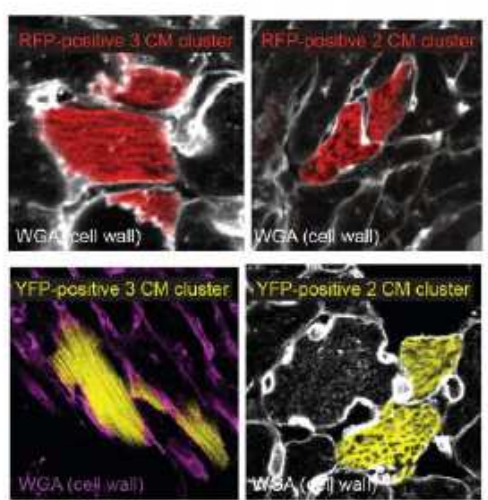

\section{Figure 1}

DUSP5 siRNA+T3 stimulates p-ERK1/2 accumulation and cardiomyocyte proliferation in young adult mice. a, The experimental protocol (top left) and representative examples of immunoblots (bottom left) and quantitative analysis (right) of p-ERK1/2 and cyclin D1 in LV cardiomyocyte lysates of 5-week-old mice treated with or without DUSP5 siRNA and T3 ( $\mathrm{n}=4$ independent biological replicates); uncropped images of immunoblots are available in Extended Data Fig. 7. Vh, vehicle. b, The experimental protocol (top) and ventricular cardiomyocyte numbers (bottom) after in vivo scrambled siRNA, scrambled siRNA+T3, DUSP5 siRNA or DUSP5 siRNA+T3 therapy ( $n=5-7$ mice per group); $* \star * P<0.001$. Comparisons were made using ANOVA with Sidak multiple comparison test $(a, b)$. c, The Confetti construct (above) and potential for expression of green (GFP), yellow (YFP), red (RFP) and cyan fluorescent protein (YFP) (below) in cardiomyocytes (below) following Cre-mediated recombination (below). $d$, The experimental protocol (top left), representative examples of monochromatic 
cardiomyocyte clusters with cell outlines delineated using wheat germ agglutinin (WGA) staining (right) and analysis (bottom left) of DUSP5 siRNA+T3 or scrambled siRNA+T3-driven monochromatic cardiomyocyte cluster formation ( $n=4-5$ mice per group). Mean values \pm s.e.m. Comparison between groups was made using a 2-tailed t-test.

a
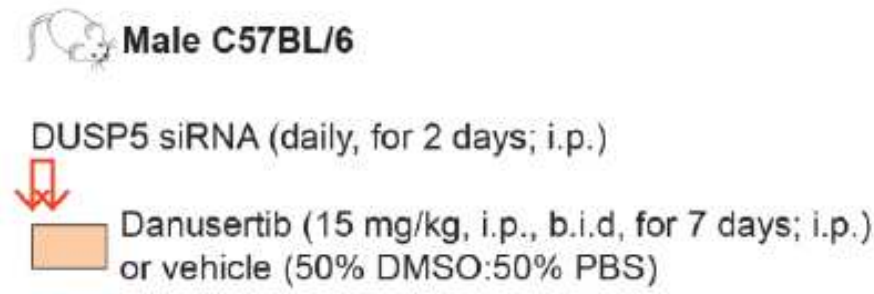

T3 (2 ng/g. daily, for 5 days; i.p.) or vehicle

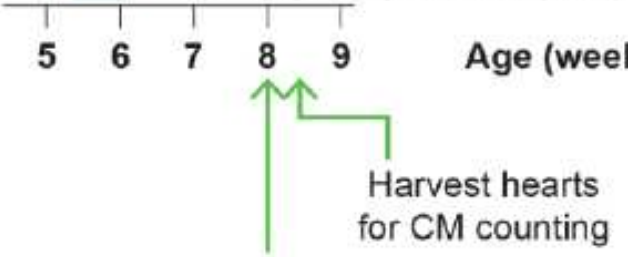

Echocardiography
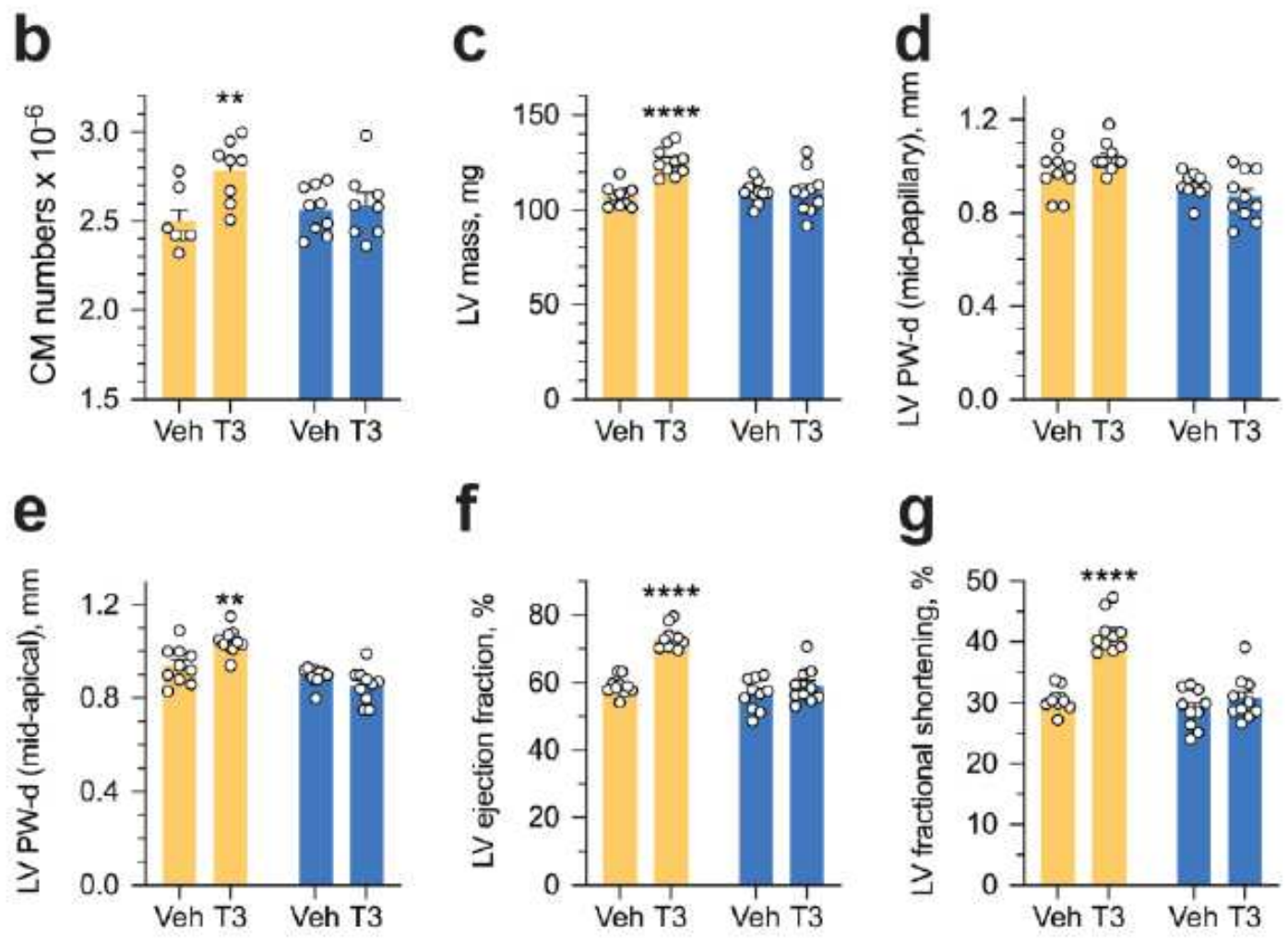

50\% DMSO:50\% PBS+DUSP5 SIRNA

Danusertib+DUSP5 siRNA

\section{Figure 2}

Cardiomyocyte proliferation drives LV growth and increases in LV contractile function after DUSP5 siRNA+T3 therapy. a, Experimental protocol for testing the effects of the cell cycle inhibitor, danusertib, on 
T3-stimulated increases in LV dimensions and contractile function in DUSP5 siRNA-treated mice. b, T3stimulated increase in ventricular cardiomyocyte numbers in DUSP5 siRNA+T3 treated mice are blocked by danusertib ( $n=6-8$ mice per group); ${ }^{*} \mathrm{P}<0.01 . \mathrm{c}-\mathrm{g}$, In vivo echocardiographic evaluation of T3stimulated changes in LV mass (c), LV PW dimensions, at diastole, at the LV mid-papillary level (d) and at the LV apex (e), LV ejection fraction ( $f$ ) and LV fractional shortening (g) in DUSP5 siRNA+T3 treated mice, with or without danusertib treatment ( $n=10$ mice per group); ${ }^{\star \star} P<0.01, * \star \star \star P<0.0001$. Mean values \pm s.e.m. Comparisons were made using ANOVA with Sidak multiple comparison test; only within group Pvalues are shown $(b-g)$. 
a

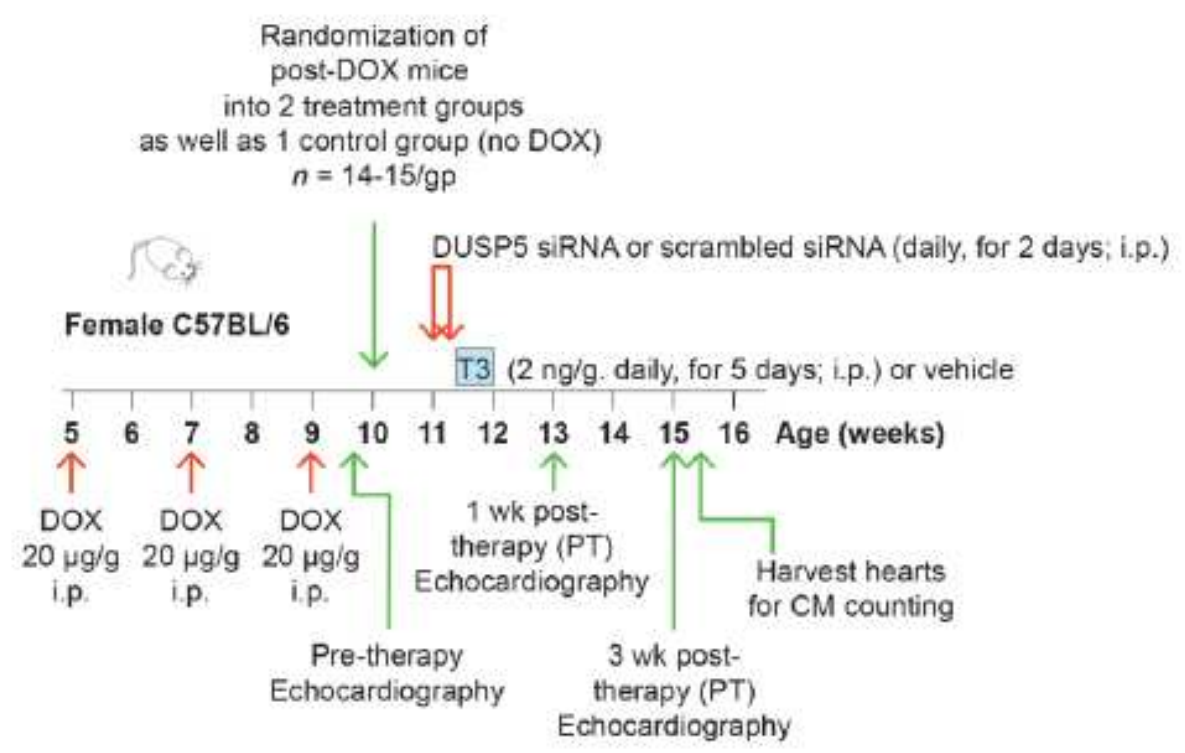

b

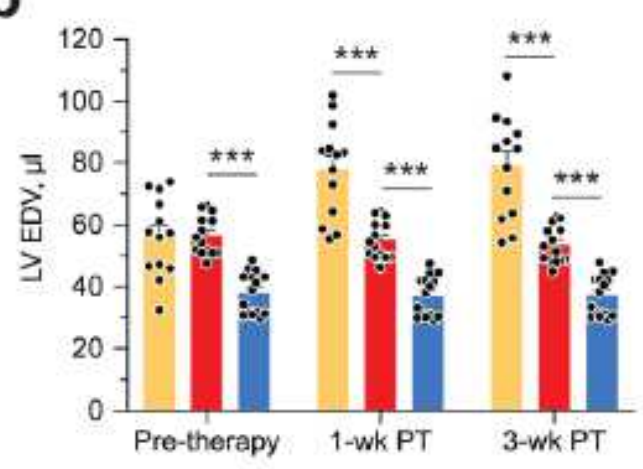

d

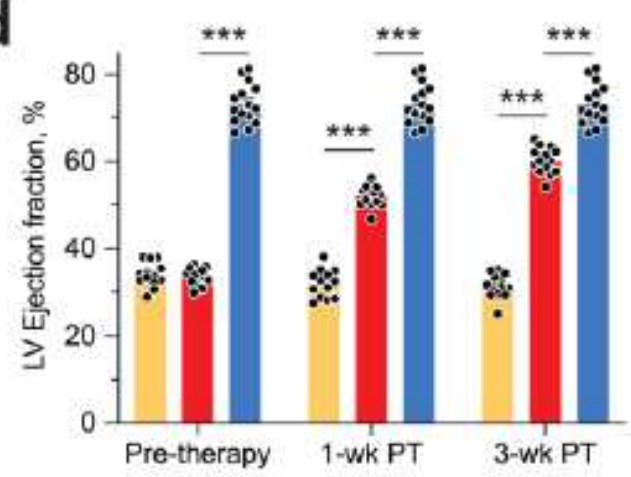

C

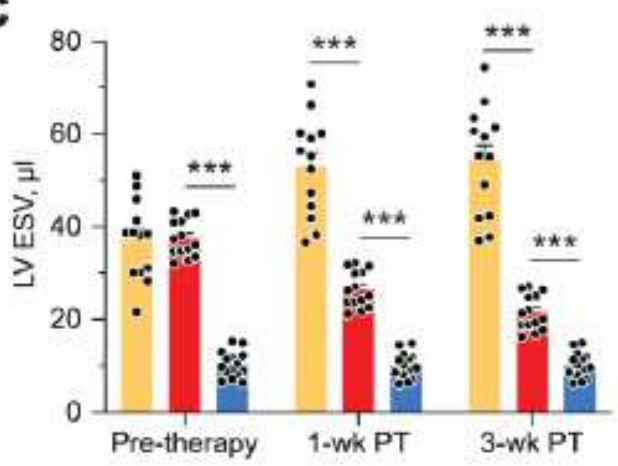

e

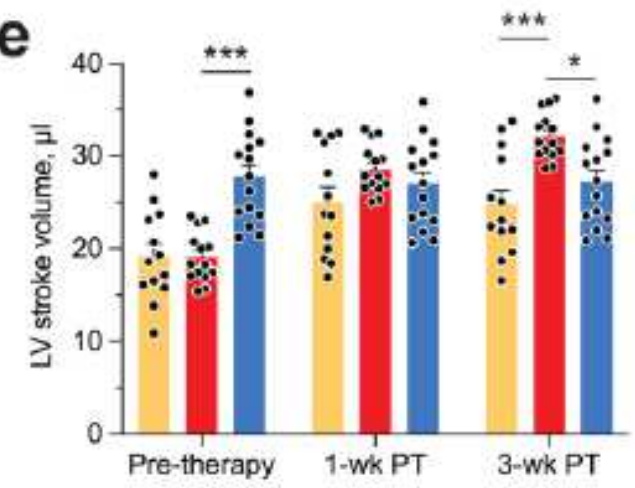

DOX+Scrambled siRNA

DOX+DUSP5 siRNA+T3

Age-matched controls

\section{Figure 3}

Transient DUSP5 siRNA+T3 therapy reverses LV dysfunction in doxorubicin-injured hearts. a, Diagram showing the experimental protocol used to create doxorubicin-induced cardiotoxicity and implementation of transient therapies with scrambled siRNA (control) or DUSP5 siRNA+T3 therapy. b-e, Repeated assessments in mice of LV end-diastolic volume (EDV) (b), end-systolic volume (ESV) (c), LV ejection fraction (d) and LV stroke volume (e) at the end of repeated doxorubicin exposure (pre-therapy) and 1- 
and 3-week after scrambled siRNA or DUSP5 siRNA+T3 therapy ( $\mathrm{n}=13-14 \mathrm{mice} / \mathrm{group})$. These temporal assessments were also made in age-matched controls that were not exposed to doxorubicin or any therapy $(n=15) .{ }^{* \star *} P<0.0001$. Data are represented as mean values \pm s.e.m. Comparisons with the doxorubicin+DUSP5 siRNA+T3 group were made using ANOVA with Sidak multiple comparison test. PT, post-therapy; DOX, doxorubicin; CM, cardiomyocyte.

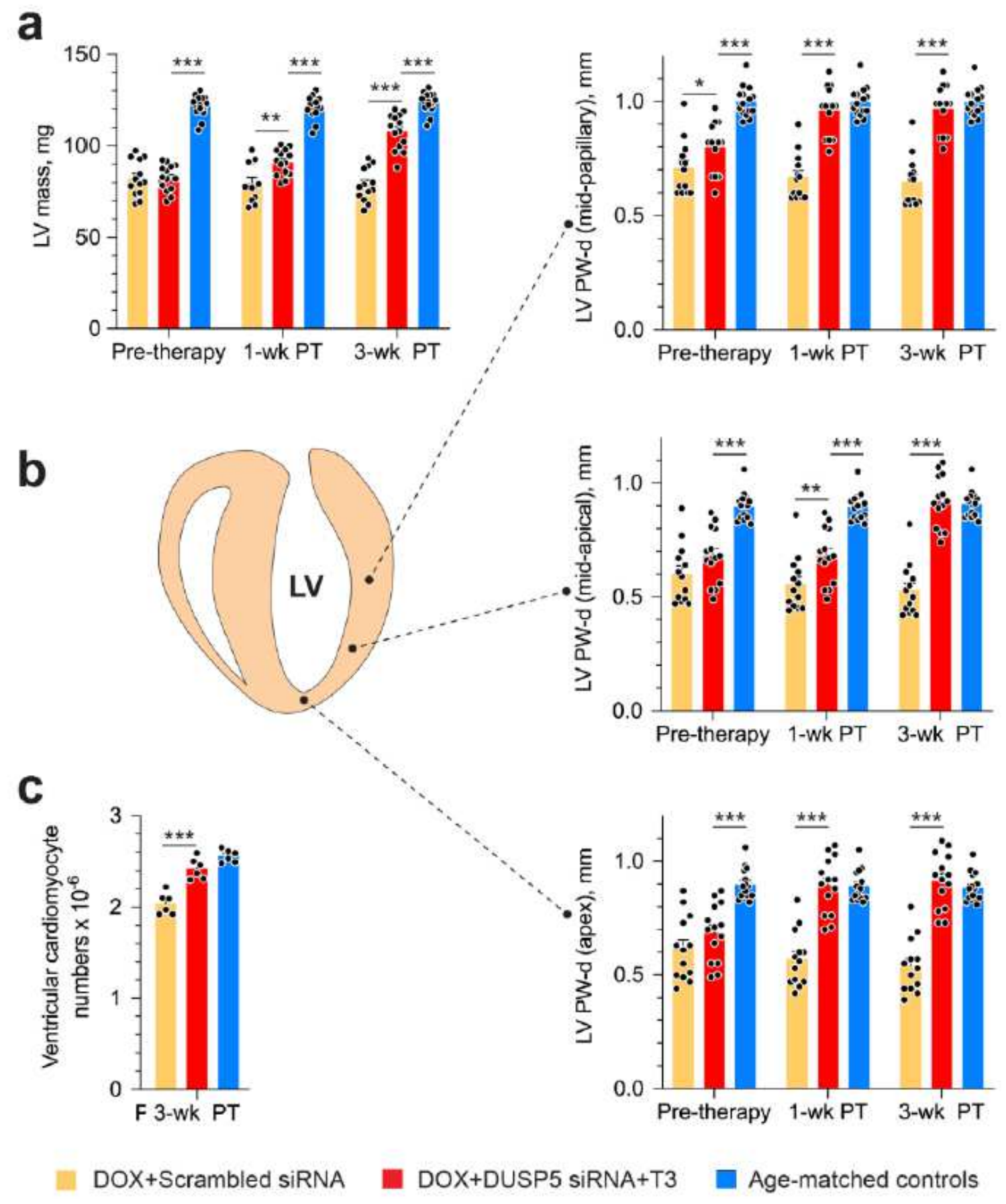

Figure 4

Transient DUSP5 siRNA+T3 therapy rebuilds cardiac muscle in mice with doxorubicin cardiomyopathy. $a, b$, Repeated assessments in mice of LV mass (a) and posterior wall (PW) dimensions at end-diastole (b) 
at the LV mid-papillary level (top) , mid-apical level (middle) and at the LV apex (bottom); assessments were made at the end of repeated doxorubicin exposure (pre-therapy) and 1- and 3-week after scrambled siRNA (control therapy) or DUSP5 siRNA+T3 therapy ( $n=13-14$ mice/group). Echocardiographic sampling sites are indicated in the diagram (middle left). Age-matched controls that were not exposed to doxorubicin or any therapy were also studied $(n=15) .{ }^{\star *} P<0.01$, ${ }^{\star \star \star} P<0.001$. Mean values \pm s.e.m. Comparisons with the doxorubicin+DUSP5 siRNA+T3 group were made using ANOVA with Sidak multiple comparison test. PT, post-therapy; DOX, doxorubicin. c, Total ventricular cardiomyocyte numbers in doxorubicin-treated mice were determined 3-weeks after scrambled siRNA or DUSP5 siRNA+T3 therapy as well as in untreated age-matched controls ( $n=6-7$ mice per group). Mean values \pm s.e.m. ${ }^{\star \star *} P<0.00$. Comparisons with the doxorubicin+DUSP5 siRNA+T3 group were made using ANOVA with Sidak multiple comparison test.
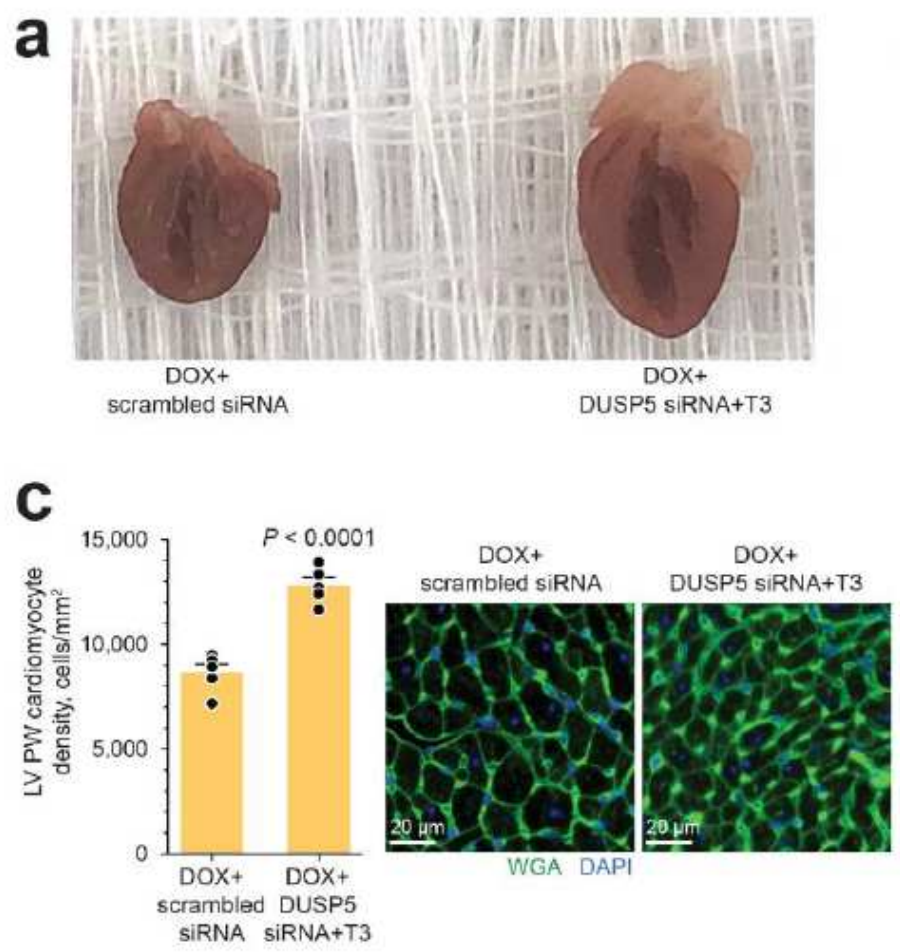
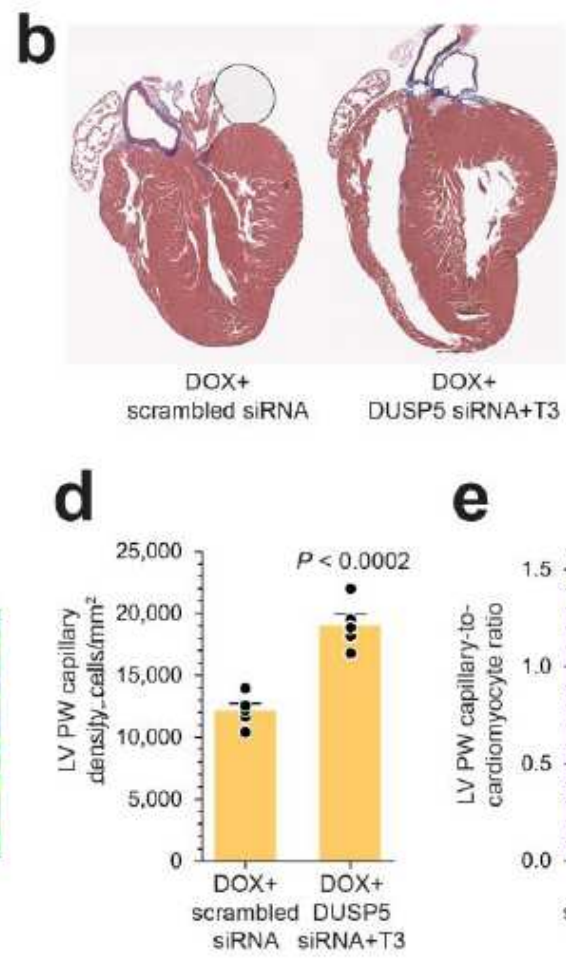

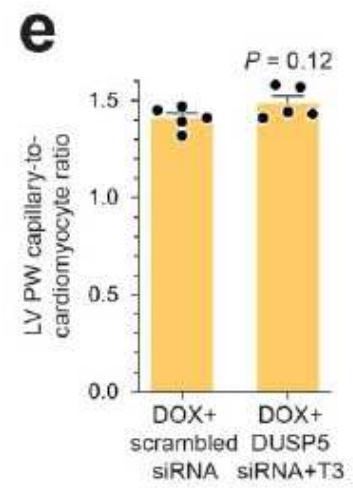

\section{Figure 5}

Gross morphology and cellular composition of doxorubicin-injured LVs following DUSP5 siRNA+T3 therapy. a,b, Gross appearance of mouse hearts cut in the longitudinal plane (a) and trichrome-stained tissue sections (b) of doxorubicin-injured hearts studied at 3-weeks following DUSP5 siRNA+T3 or control scrambled siRNA therapy. c,d, LV PW cardiomyocyte (c) and capillary densities (d) were assessed after staining with wheat-germ agglutinin (WGA) and 4',6-diamidino-2-phenylindole (DAPI) to identify cell borders and nuclei, respectively. Representative images are shown in c (right). Bars, $20 \mu \mathrm{m}$.

Determinations were made using tissue sections from the LV mid-papillary region. e, Capillary-to 
cardiomyocyte ratios. Comparison between groups were made using a 2-tailed $t$-test $(n=5$ mice per group in c-e). Data are represented as mean values \pm s.e.m.

\section{Supplementary Files}

This is a list of supplementary files associated with this preprint. Click to download.

- ExtendedDataVideo1.mp4

- ExtendedDataVideo2.mp4

- OnlineMethodsTAN.pdf 